\title{
The Impact of Coronavirus Disease (COVID-19) on Patients Visiting Outpatient Urology Clinic in a Nigerian Tertiary Hospital: A Cross Sectional Study
}

\author{
Ehiremhen Ozah $^{1, *}$, Eshiobo Irekpita ${ }^{2}$ \\ ${ }^{1}$ Urology Unit, Department of Surgery, Irrua Specialist Teaching Hospital, Irrua, Nigeria \\ ${ }^{2}$ Department of Surgery, Urology division, Ambrose Alli University, Ekpoma, Nigeria
}

Email address:

e.ozah@yahoo.com (E. Ozah), ieshiobo@yahoo.com (E. Irekpita)

${ }^{*}$ Corresponding author

To cite this article:

Ehiremhen Ozah, Eshiobo Irekpita. The Impact of Coronavirus Disease (COVID-19) on Patients Visiting Outpatient Urology Clinic in a Nigerian Tertiary Hospital: A Cross Sectional Study. Central African Journal of Public Health. Vol. 7, No. 4, 2021, pp. $182-188$. doi: 10.11648/j.cajph.20210704.15

Received: June 16, 2021; Accepted: June 28, 2021; Published: July 2, 2021

\begin{abstract}
Background: The coronavirus pandemic had a major impact on health care service delivery globally. Work force in the health care sector and resources were focused on the critically ill from Coronavirus disease (COVID-19) following the huge number of patients contracting this rapidly spreading virus. All medical specialties not directly involved in treatment of COVID-19 witnessed interruption in services, urologic care was not spared. Objective: This study aims to assess the impact of coronavirus Disease -19 (COVID-19) on patients visiting urology outpatient clinic at Irrua Specialist Teaching Hospital. Methods: It was a prospective cross-sectional study conducted to assess impact of COVID-19 on patients visiting outpatient urology clinic. All consecutive patients aged 18 years and above who consented were recruited. Continuous data like age were presented in mean and standard deviation, while categorical data were presented in percentages. Association between dependent and independent variables were determined using Pearson chi square or fisher's exact where appropriate while predictors of impact were assessed using multivariate logistic regression. P-value set as 0.05 . Results: A total of 154 respondents participated. Mean age was 61.9 years. Males constitute $90.3 \%$ of respondent. Only $6 \%$ of participants felt negative impact of COVID-19 on urology care. Age of respondent was the only determinant of impact of COVID-19 (pvalue $=0.014)$. There were no predictors of impact amongst socio demographic characteristics in a multivariate logistic regression. In the study $48.1 \%, 16.2 \%$ and $12.3 \%$ of participants suggested the use of phone calls, home visits and telemedicine as means of consultation to improve outpatient urology care and reduce the spread of COVID-19. Conclusions: The impact of COVID-19 on outpatients urology care was minimal at Irrua Specialist Teaching Hospital. Age was a determinant of impact. Improvement in access to telemedicine and phone calls were suggested as measures to further improve urology care while efforts are put in place to prevent spread of disease and achieve cure during the pandemic.
\end{abstract}

Keywords: COVID-19, Impact, ISTH, Outpatient, Urology

\section{Introduction}

The novel coronavirus was first reported in Wuhan City, Hubei Province of China, in December 2019 and has spread across over 213 countries and territories around the world [1, 2]. It was declared a Public health emergency of international concern on February 11, 2020. The disease was named coronavirus disease (COVID-19) January 30, 2020, while it was declared a pandemic by the World Health Organization on March 11, 2020 [3].

Coronavirus is a family of enveloped single stranded RNA viruses that cause respiratory, enteric, hepatic and neurologic disease $[4,5]$. Human coronavirus $(\mathrm{CoV})$ infections are caused by $\alpha-$ and $\beta$-CoVs $[4,5]$. Severe acute respiratory syndrome coronavirus (SARS-CoV) and middle east respiratory syndrome coronavirus (MERS-CoV) are members of $\beta-\mathrm{CoV}$ family [4] and share $79.5 \%$ and $50 \%$ of their genomes with SARS-CoV 2 respectively $[6,7]$. 
Coronavirus disease (COVID-19) is highly contagious through SARS-CoV-2 virus containing respiratory droplets of infected persons, this can be deposited on oral, nasal or ocular mucous membranes of nearby persons. The virus can be transmitted by human to human contact or contact with infected objects [8]. The incubation period ranges from 2-14 days [9].

The infection of SARS-CoV2 can be asymptomatic or symptomatic producing symptoms like fever (98\%), fatigue (70\%), dry cough $(60 \%)$, anosmia. These symptoms can progress to dyspnea in $30 \%$ of the patients [10]. Nonrespiratory symptoms like diarrhea and nausea occur in $10 \%$ of cases [10]. About 30\% of patients are asymptomatic, 55\% have mild to moderate symptom and $15 \%$ severe to critical symptoms $[9,11]$. As at 3 May 2021, over 150 million cases have been recorded with over 3 million deaths [12]. The burden of the disease varies across different countries. In Nigeria, greater than 165,000 cases have been recorded with about 2000 deaths [13].

The disease toll has caused a change in medical practice and the way of life throughout the world [14], this has signaled the use of non-pharmacological measures to curtail the disease. This include physical distancing, hand hygiene and use of face masks. The COVID-19 pandemic has significantly impacted on wide array of health services globally particularly in low and middle income countries [1517], Urology practice was not spared, as a large sample international survey [18], revealed diminished urological services with COVID-19 outbreak resulting in delay of 8 weeks in outpatient clinics, outpatient investigation and procedures and urologic surgeries in $28 \%, 30 \%$ and $31 \%$ of cases respectively, a reduction in patient volume was also noted in outpatient clinic during this study due probably to fear of contracting the infection and the lockdown imposed by government in Nigeria during this period. Therefore, it is pertinent to estimate the degree of impact of COVID-19 in on urological services, in a bid to re organize health care strategy for better outcome.

Therefore, this study aims to assess the impact of COVID19 on patients visiting urology outpatient clinic at Irrua Specialist Teaching Hospital (ISTH), Irrua, Edo State.

\section{Methods}

\subsection{Study Setting, Design}

This is a prospective cross sectional descriptive study conducted using a self-administered structured questionnaire. The study was carried out at Irrua specialist Teaching Hospital, located at km 87 Benin- Auchi road, Irrua, Edo state. A 400 bed capacity hospital established as tertiary health care delivery center, offering a range of specialist urology services. It is also a designated infectious disease referral Centre. A total of 154 participants were involved at all level of this study.

\subsection{Data Collection}

A pretested questionnaire which included demographic variables (Age, sex, Religion, Ethnicity, marital status, occupation and level of education) this represented independent variables, 11 variables on impact of COVID-19 on urologic practice were developed. A scoring method was developed by author to assess overall impact of COVID-19 which was the dependent variable, a minimum score of $50 \%$ was considered as having minimal or no impact on participants.

Data collected by using structured questionnaire which was self-administered by patients visiting outpatient, Urology clinic who gave written consent and met the inclusion criteria. To ensure confidentiality the names of participants and case note numbers were excluded from the questionnaire.

\subsection{Study Population}

Participants include all consecutive patients aged 18 and above presenting to the outpatient Urology clinic during the period of the study. The exclusion criteria was failure to consent and all these below the age of 18 years.

\subsection{Data Analysis}

Statistical analysis was carried out using IBM SPSS (statistical package for the social sciences), software version 21. Numerical data, normally distributed such as age (years) were expressed in mean \pm standard deviation. Categorical data such as (sex, marital status, level of education, religion, ethnic group, dependent variables) were expressed as frequencies and percentages. A scoring system developed to assess overall impact. Association between independent variables (social demographic characteristics) and dependent variables (impact) were assessed using fisher's exact in a bivariate analysis; While predictor of dependent variables (socio-demographic characteristics) were assessed using logistic regression model in a multivariate analysis. The level of significance was set at $\mathrm{p}<0.05$.

\subsection{Ethical Approval}

All eligible participants were informed about the aims of the study, consent was signed before participation. The protocols of the study were approved by the Hospital research and ethics committee (HREC) of Irrua specialist Teaching Hospital with protocol number: ISTH/HREC/20200506/074. Participants were informed of their right to withdraw from study at any time they wish without any consequence.

\section{Results}

\subsection{Socio-demographic Characteristics of Participants}

The study was carried out between June and November, 2020. Following ethical approval and consent from the participants, one hundred and fifty four consecutive patients presenting in the outpatient urology clinic within this period who gave consent participated. The mean age of participants was $61.9 \pm 12.9$ (Standard Deviation) years (Table 1). 
Table 1. Age distribution of participants in decades.

\begin{tabular}{lll}
\hline Age (years) & Frequency & Percent \\
\hline$<20$ & 1 & 0.6 \\
$20-29$ & 4 & 2.6 \\
$30-39$ & 6 & 3.9 \\
$40-49$ & 7 & 4.5 \\
$50-59$ & 23 & 14.9 \\
$60-69$ & 77 & 50.0 \\
$70-79$ & 28 & 18.2 \\
$\geq 80$ & 8 & 5.2 \\
Total & 154 & 100.0 \\
\hline
\end{tabular}

Of the one hundred and fifty four participants, $139(90.3 \%)$ were males while $15(9.7 \%)$ were females. One hundred and thirty $(84.4 \%)$ participants were married while thirteen $(8.4 \%)$ were single.

Majority of the participants $(57 \%)$ had tertiary level of education (Figure 1).

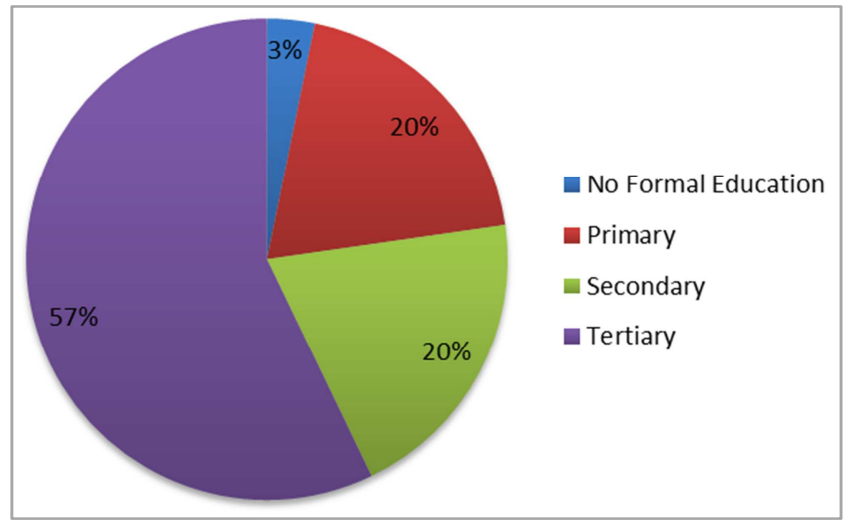

Figure 1. Educational level of participants.

Using the international standard classification of skill level, $23(14.9 \%)$ participants had occupational skill level 1, 34 (22.1\%) had occupational skill level 2, 27 (17.5\%) had level 3 and $16(10.4 \%)$ had level 4 while $54(31.5 \%)$ did not provide information on this. One hundred and twenty-nine (83.8\%) respondents were Christians and twenty (13.0\%) respondents were Muslims while others either practiced traditional African religion $(1.9 \%)$ or were atheists $(1.3 \%)$. Forty-seven $(30.5 \%)$ were Esan while eleven (30.5\%) were Benin.

\subsection{Impact of COVID-19}

Ninety-five (61.7\%) respondents agreed COVID-19 have not affected their desire to attend hospital. One hundred and six $(68.8 \%)$ respondents agreed that their clinic appointment has not changed because of COVID-19. Ninety (58.4\%) respondents agreed COVID-19 has not altered the way they attend the hospital. One hundred and thirty-nine $(90.3 \%)$ respondents have not bought drugs without seeing their urologist. One hundred and fifty $(97.4 \%)$ respondents have not sought alternative medicine for urological condition. Seventy-two (46.8\%) respondents condition has not changed since the advent of COVID-19. One hundred and forty $(90.0 \%)$ respondents have not had a postponement in procedure. One hundred and one
(65.6\%) respondents did not developed any urological emergency during COVID-19. (Table 2).

Table 2. Impact of COVID-19.

\begin{tabular}{|c|c|c|}
\hline Variable & Frequency $(n=154)$ & Percent \\
\hline \multicolumn{3}{|c|}{ COVID-19 affected your desire to attend hospital } \\
\hline Not at all & 95 & 61.7 \\
\hline Not much & 36 & 23.4 \\
\hline Seriously & 14 & 9.1 \\
\hline Very seriously & 9 & 5.8 \\
\hline \multicolumn{3}{|c|}{ How did COVID-19 impact on interval between clinic appointment } \\
\hline More & 13 & 8.4 \\
\hline Unchanged & 106 & 68.8 \\
\hline Reduced & 35 & 22.7 \\
\hline \multicolumn{3}{|c|}{ Do you think COVID-19 has altered the way you are attended to in the hospita } \\
\hline Not at all & 90 & 58.4 \\
\hline Minimally & 30 & 19.5 \\
\hline Seriously & 24 & 15.6 \\
\hline Very seriously & 10 & 6.5 \\
\hline \multicolumn{3}{|c|}{ Have you been buying drugs outside without seeing your urologist } \\
\hline Yes & 15 & 9.7 \\
\hline No & 139 & 90.3 \\
\hline \multicolumn{3}{|c|}{ Did you seek alternative medicine for urological condition } \\
\hline Yes & 4 & 2.6 \\
\hline No & 150 & 97.4 \\
\hline \multicolumn{3}{|c|}{ How has your condition fared since advent of COVID-19 } \\
\hline Much improved & 20 & 13.0 \\
\hline Improved & 56 & 36.4 \\
\hline No change & 72 & 46.8 \\
\hline Worse & 6 & 3.9 \\
\hline \multicolumn{3}{|c|}{ Awaiting any procedure which has been postponed } \\
\hline Yes & 14 & 9.1 \\
\hline No & 140 & 90.9 \\
\hline \multicolumn{3}{|c|}{ Did you develop any urological emergency during COVID-19 } \\
\hline Yes & 53 & 34.4 \\
\hline No & 101 & 65.6 \\
\hline
\end{tabular}

\subsection{Impact Score of COVID-19}

In Majority (94\%) of the respondents Urology care was not impacted by COVID-19. (Figure 2)

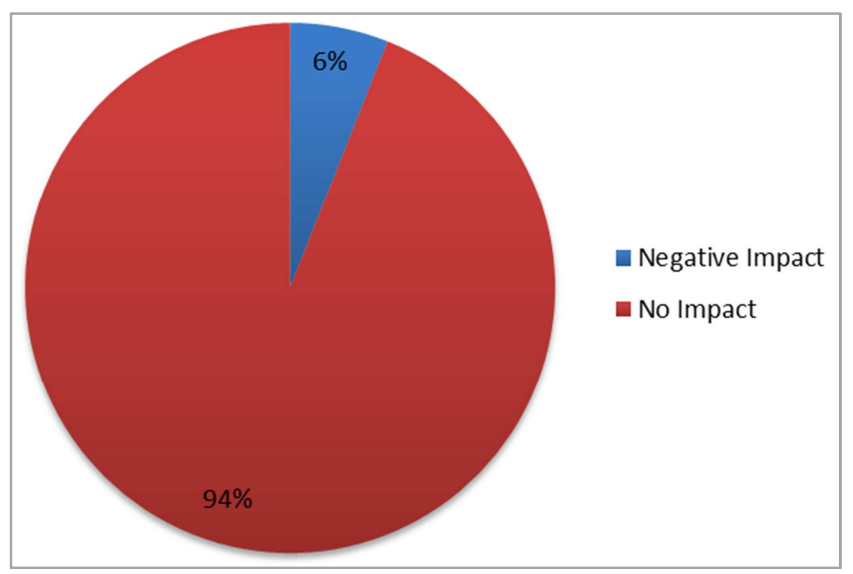

Figure 2. Impact Score of COVID-19. 


\subsection{Association Between Socio-demographic and Impact of COVID-19}

Age of respondent was the only determinant of impact of COVID-19 (P-value $=0.014)$.

Using Fisher's exact to determine association between age and impact of COVID-19, there was a statistically significant association $(\mathrm{p}=0.014)$, while marital status, level of education, occupational skill level, religion and ethnicity were statistically insignificant with p-values of $0.081,0.322,0.555$, 0.733 and 0.270 respectively. Sex was also not a determinant of impact using chi-squared with a p-value of 0.599 . (Table 3 )

Table 3. Association between socio-demographic and impact of COVID-19.

\begin{tabular}{|c|c|c|c|c|}
\hline \multirow{2}{*}{ Variable } & \multicolumn{2}{|c|}{ Frequency n (\%) } & \multirow{2}{*}{ Test statistics } & \multirow{2}{*}{ P value } \\
\hline & No Impact & Negative Impact & & \\
\hline \multicolumn{5}{|l|}{ Age of respondent } \\
\hline$<20$ & $0(0.0)$ & $1(10.0)$ & Fisher's Exact=15.344 & 0.014 \\
\hline $20-29$ & $3(2.1)$ & $1(10.0)$ & & \\
\hline $30-39$ & $5(3.5)$ & $1(10.0)$ & & \\
\hline $40-49$ & $7(4.9)$ & $0(0.0)$ & & \\
\hline $50-59$ & $22(15.3)$ & $1(10.0)$ & & \\
\hline $60-69$ & $74(51.4)$ & $3(30.0)$ & & \\
\hline $70-79$ & $27(18.8)$ & $1(10.0)$ & & \\
\hline$\geq 80$ & $6(4.2)$ & $2(20.0)$ & & \\
\hline \multicolumn{5}{|l|}{ Sex } \\
\hline Male & $129(89.6)$ & $10(100.0)$ & $\chi^{2}=1.154$ & 0.599 \\
\hline Female & $15(10.4)$ & $0(0.0)$ & & \\
\hline \multicolumn{5}{|l|}{ Marital status } \\
\hline Single & $10(6.9)$ & $3(30.0)$ & Fisher's Exact=4.989 & 0.081 \\
\hline Married & $123(85.4)$ & $7(70.0)$ & & \\
\hline Widowed & $11(7.6)$ & $0(0.0)$ & & \\
\hline \multicolumn{5}{|l|}{ Level of education } \\
\hline No formal education & $4(2.8)$ & $1(10.0)$ & Fisher's Exact=3.129 & 0.322 \\
\hline Primary & $29(20.1)$ & $1(10.0)$ & & \\
\hline Secondary & $28(19.4)$ & $3(30.0)$ & & \\
\hline Tertiary & $83(57.6)$ & $5(50.0)$ & & \\
\hline \multicolumn{5}{|l|}{ Occupational level } \\
\hline Skill level 1 & $22(23.4)$ & $1(16.7)$ & Fisher's Exact=2.165 & 0.555 \\
\hline Skill level 2 & $33(35.1)$ & $1(16.7)$ & & \\
\hline Skill level 3 & $25(26.6)$ & $2(33.3)$ & & \\
\hline Skill level 4 & $14(14.9)$ & $2(33.3)$ & & \\
\hline \multicolumn{5}{|l|}{ Religion } \\
\hline Christian & $121(84.0)$ & $8(80.0)$ & Fisher's Exact=1.615 & 0.733 \\
\hline Muslim & $18(12.5)$ & $2(20.0)$ & & \\
\hline African traditional religion & $3(2.1)$ & $0(0.0)$ & & \\
\hline Atheist & $2(1.4)$ & $0(0.0)$ & & \\
\hline \multicolumn{5}{|l|}{ Ethnic group } \\
\hline Esan & $44(44.4)$ & $3(42.9)$ & Fisher's Exact=4.212 & 0.270 \\
\hline Bini & $10(10.1)$ & $1(14.3)$ & & \\
\hline Etsako & $7(7.1)$ & $2(28.6)$ & & \\
\hline Delta & $6(6.1)$ & $0(0.0)$ & & \\
\hline Others & $32(32.3)$ & $1(14.3)$ & & \\
\hline
\end{tabular}

\subsection{Predictors of Impact of COVID-19}

There was no predictor of impact of COVID-19 among the socio-demographic characteristics. Using a multivariate logistics model, there were no predictors of impact using age of respondents, sex, marital status, level of education, occupational skill level with p-values more than 0.05 , therefore statistically not significant (Table 4 ). 
Table 4. Predictors of impact of COVID-19.

\begin{tabular}{|c|c|c|c|c|c|}
\hline \multirow{2}{*}{ Variables } & \multirow{2}{*}{ B (regression coefficient) } & \multirow{2}{*}{$p$ value } & \multirow{2}{*}{ Odds ratio } & \multicolumn{2}{|c|}{ 95\% C. I. for Odds ratio } \\
\hline & & & & Lower & Upper \\
\hline Age of respondents & -0.019 & 0.456 & 0.981 & 0.934 & 1.031 \\
\hline \multicolumn{6}{|l|}{ Sex } \\
\hline Male & & & 1 & & \\
\hline Female & -0.138 & 0.903 & 0.871 & 0.096 & 7.934 \\
\hline \multicolumn{6}{|l|}{ Marital status } \\
\hline Single & & & 1 & & \\
\hline Married & -1.470 & 0.112 & 0.230 & 0.037 & 1.411 \\
\hline Widowed & -0.767 & 0.590 & 0.465 & 0.029 & 7.533 \\
\hline \multicolumn{6}{|l|}{ Level of education } \\
\hline No informal & & & 1 & & \\
\hline Primary & -1.723 & 0.249 & 0.179 & 0.010 & 3.346 \\
\hline Secondary & -0.642 & 0.618 & 0.536 & 0.046 & 6.240 \\
\hline Tertiary & -1.200 & 0.313 & 0.301 & 0.029 & 3.093 \\
\hline \multicolumn{6}{|l|}{ Occupation } \\
\hline Skill level 1 & & & 1 & & \\
\hline Skill level 2 & -0.405 & 0.622 & 0.667 & 0.040 & 11.227 \\
\hline Skill level 3 & 0.565 & 0.778 & 1.760 & 0.149 & 20.764 \\
\hline Skill level 4 & 1.145 & 0.653 & 3.143 & 0.260 & 37.991 \\
\hline
\end{tabular}

\subsection{Ways of Improving Outpatient Care by Participants}

A greater proportion of the respondents $74(48.1 \%)$ suggested phone calls as a means of consultation while 33 (21.4\%) participants there were missing data on ways of improving outpatient care during the pandemic. (Table 5).

Table 5. Ways of improving outpatient care by participants.

\begin{tabular}{lll}
\hline Variable & Frequency & Percent \\
\hline Telemedicine & 19 & 12.3 \\
Phone calls & 74 & 48.1 \\
Home visit & 25 & 16.2 \\
Others & 4 & 2.6 \\
\hline
\end{tabular}

*Multiple response

Others include: Reassuring patients, prompt response to outpatients, reduce duration of consultation

\section{Discussion}

The global pandemic led many urologists to make unprecedented decisions for striking a balance between providing optimal high quality urological care to their patients while reducing the risk of spreading infections among patients and health care workers. Hospitals that cater for COVID-19 patients have to temporarily suspend effective surgeries to cope with the devastating surge in COVID-19 cases; this had a toll on out patients and in-patient, urology care $[19,20]$. Health care system witnessed reorganization and restructuring of resources and personnel. Out patients visit were reduced to only these deemed absolutely necessary, while others adopted telemedicine platform [21]. Dubin et al [22] found lack of technological comprehension, patient's lack of access to required technology and reimbursement concern as barriers to use of telemedicine. A reduction was noted in number of patients attending the outpatient clinic, which may not have been unconnected to the fear of the pandemic amongst patients and the lock down imposed across the country during this period.

Never the less, in this study overall impact of COVID-19 on the urologic care was minimal, only $6 \%$ of respondent subscribed to the fact that health care was negatively impacted. A good number of respondents still had the desire to attend clinic. Over $68 \%$ of participants claim that clinic interval was unchanged. Ninety percent of respondents did not have cause to procure drug without consulting their Urologist. Amongst patient who participated in this study only $2.6 \%$ sought alternative medicine for their urologic condition while $9.1 \%$ had their urologic procedures postponed, reasons advanced for this were that most were non oncological cases, procedures that did not require intervention for organ threatening or life threatening cases. This findings contrast with findings in a study [18] that revealed substantial disruption in urological care and an overall reduction in outpatient clinic volume, however, in their study, the greatest reduction in outpatient clinic volume was observed for benign and non-urgent conditions particularly in areas with highest COVID-19 cases. In our study the reason for the low impact maybe multifaceted which include, our center being an infectious disease designated referral center, there was low level of fear and anxiety amongst health care workers who were already exposed to infectious disease prevention and control measures. Nigeria did not witness exponential surge in cases and death, as witnessed in most parts of the world.

Age was the only socio demographic characteristics that determined impact of COVID-19 on health care seeking behavior in our respondents and it was statistically significant ( $p$-value $=0.014$ fisher's exact) this may be due to the fact that health seeking behavior is more established in the ageing population and the mean age of the study population was 61.9 years. Multivariate logistic regression 
model however, revealed no predictor of impact of COVID19 among the socio demographic characteristics. Consequently, respondents $(48.1 \%)$ suggested use of telephones to improve outpatient care this will keep patients at home reducing the chances of exposure to COVID-19. In this study $16.2 \%$ recommended home visit by the Urologist. Use of telemedicine, was recommended by $12.3 \%$ of participants. In a previous study [23] acceptance for telehealth for hospital consultations have been explored and it clearly demonstrated a potential for improving urologic care amongst, younger patients, these with higher education level, previous exposure to video conferencing tools, these travelling longer distance and days missed from work. Furthermore, telehealth has also being shown to reduce overall cost to patients [24], although barriers to achieving this feat has been highlighted in a study by Dubin et al [22], in their study reason adduced includes lack of technology, patients access and reimbursement concerns, perhaps this also applies in Nigeria. About $2.6 \%$ of participants recommended reduction in waiting time during consultations to reduce the risk of exposure.

\section{Limitations and Strength of the Study}

Strength of this study was hinged on the fact that it was the first in the instituition and the locality to assess the impact on patients rather than health workers and facilities. Limitations of this study was the noticeable reduction in number of patients who attended the outpatient urology clinic, during the period of the study, who were not part of this study, their participation could have impacted on the outcome of this study. Furthermore, scoring system used for assessment of overall impact had not being previously validated.

\section{Conclusion}

The study revealed that impact of COVID-19 on outpatient urology care was not marked in at Irrua Specialist Teaching Hospital, amongst patients who presented to clinic, despite a reduction in volume of patients who sought urology care, while interval of visit and procedures remain unchanged. Age impacted on health seeking behavior, however no socio demographic characteristics was a predictor of impact.

Improvement in access to technology, by provision of means of communication through telehealth and telephony between patients and physicians were suggested by participants as measures that will further improve urology care and health care in general during the COVID-19 pandemic and in future practice.

It is recommended that a more holistic study involving all patients including these who have defaulted follow up because of the pandemic will be more representative. Therefore, future research on this subject would be community based as this would add more statistical power to outcome.

\section{Declaration}

\section{Consent for Publication}

Not applicable.

\section{Availability of Data and Materials}

The datasets used and/or analysed during this study are available from the corresponding author on reasonable request.

\section{Competing Interest}

The authors declare that they have no competing interests.

\section{Funding}

No funding was received by authors at any stage of this study.

\section{References}

[1] World Health Organisation, Coronavirus disease (COVID-19) Pandemic. Geneva, Switzerland: World Health Organization; April 12, 2020.

[2] Bogoch II Watts A, Thomas-Bachl: A, Huber C, Kraemer MUG, Khan K. Pneumonia of unknown aetiology in Wuhan, China: potential for international spread via commercial air travel. J Travel Med 2020; 27: taaa 008.

[3] Worldometer, COVID-19 coronavirus pandemic. Worldometer Web site; April 12, 2020.

[4] Weiss SR, Leibowitz JL. Coronavirus pathogenesis. Adv virus Res. 2011; 81: 85-164.

[5] De Wilde AH, Snijder EJ, Kikkert M, Van Hemert MJ. Host factors in coronavirus Replication. In Roles of Host Gene and Non-coding RNA Expression in Virus Infection; Tripp RA, Tompkins SM, Chan Switzerland, Springer International Publishing 2018; pp. 1-42.

[6] Jin Y, Yang H, J; W, Wu, W, ChengS, Zhang W, et al. Virology, Epidemiology, pathogenesis and control of COVID19 Viruses. 2020; 12: 372-389.

[7] Lin L, Lu L, Cao W, Li T. Hypothesis for potential pathogenesis of SARS-CoV-2 infection. A review of immune changes in patients with viral pneumonia Emerg Microbes Infect, 2020; 9: 727-732.

[8] Yang Y, Peng F, Wang R, Guan K, Jian T, Xu G, et al. the deadly coronaviruses: the 2003 SARS pandemic and the 2020 novel coronavirus epidemic in China. J Autoimmun, 2020; 109: 102434. Erratum in: J Autoimmun. 2020; 111: 102487.

[9] Lauer SA, Grantz KH, Bi Q, Jones FK, Zheng Q, Meredith $\mathrm{HR}$, et al. the incubation period of coronavirus Disease 2019 (COVID-19) from Publicly Reported Confirmed Cases: Estimation and Application. Ann Intern Med. 2020 May 5; 172 (9): 577-582 doi 10.7326/m20-0504. Epub 2020 Mar 10.

[10] Wang D, Hu B, Hu C, Zhu F, Liu X, Zhang J et al. clinical Characteristics of 138 Hospitalized Patients with 2019 Novel coronavirus- infected Pneumonia in Wuhan, China. JAMA, 2020; 323: 1061-1069. 
[11] Verity R, Okell LC, Dorigatti I, Winskill P, Whiltaker C, Imai N, et al. Estimates of the severity of coronavirus disease 2019: a model-based analysis. Lancet Infect Dis. 2020 (6): 669-677 doi 10.1016/51473-3099(20)30243-7 Epub 2020.

[12] World Health Organisation Coronavirus disease (COVID-19) Situation reports. Available from: https://www.who.int/emergencies/disease/novel-coronavirus2019/situation-reports. Accessed May 3, 2021.

[13] Worldometer, COVID-19 coronavirus pandemic. Worldometer Website; May4, 2021.

[14] Sampaio FJB, Reflections on the COVID-19 Pandemic. Int Braz J Urol. 2020; 46: 499-500.

[15] World Health Organization. COVID-19 Significantly Impacts Health Services for Non-communicable Diseases; 2020. Available From http://www.who.int/news-room/detail/01-062020-covid-19-significantly-impacts-health-services-fornoncommunicable-diseases [accessed on 2020 June 19].

[16] Riley T, Sully E, Ahmed A, Biddelecom A. Estimates of the Potential Impact of the COVID-19 Pandemic on sexual and reproductive Health In Low and Middle-Income Countries. Int Perspect Sex Reprod Health 2020; 46: $73-76$ doi: $10.1363 / 4609020$.

[17] The Global Fund. Global Fund Survey: Major of HIV, TB and Malaria Programs Face Disruptions as a result of COVID-19; 2020 Available from: https://www. Theglobal fund.org/en/covid-19/news/2020-06-17-global-fund-surveymajority-of-hiv-tb-and-malaria-programs-face-May07.
[18] Teoh JY, Ong WLK, Gonazalez -Padilla D, et al. A Global Survey on the Impact of COVID-19 on Urological Services. Eur Urol, 2020; 78 (2): 265-275. Doi: 10.1016/j.eururo, 2020.05.02.

[19] Chan MC, Yeo SEK, Chong YL, Lee YM. Stepping Forward: Urologists efforts during the COVID-19 outbreak in Singapore. Eur Urol. 2020 Jul; 78 (1): 38-39 doi: 10.1016/j.euro.2020.03.004.

[20] Norwroozi A, Amini E. Urology Practice in the time of COVID-19. Urol J. 2020 May 16; 17 (3): 326 doi 10.22037/uj.vOiO.6065.

[21] Cacciamani GE, Shah M, Yip W, Abreu A, Park D, Fuchs G. Impact of COVID-19 on the urology service in United States: Perspectives and strategies to face a pandemic. Int Braz J. Urol. 2020 Jul; 46 (Suppl. 1): 207-214. doi: 10.1590/51677-5538.

[22] Dubin JM, Wyant WA, Balaji NC, Ong WL, Kettache RH, Haffaf $M$ et al. Telemedicine Usage Among Urologist During the COVID-19 Pandemic: Cross Sectional Study. J Med Internet Res. 2020 Nov 5; 22 (11): e21875.

[23] Viers BR, Pruthi S, Rivera ME, O' Neil DA, Gardener MR, Jenkins SM et al. Are patients willing to engage in Telemedicine for their care: A Survey of Preuse Perceptions and Acceptance of Remote Video Visits in A urological Patient Population. Urology. 2015; 85: 1233-1239.

[24] Viers BR, Lightner DJ, Rivera ME, Tollefson MK, Boorjian SA, Karnes RJ et al. Efficiency, satisfaction and costs for remote video visits following radical prostatectomy: a randomized controlled trial. Eur Urol. 2015; 68: 729-735. 